\title{
Ultrafast release and capture of carriers in InGaAs/GaAs quantum dots observed by time-resolved terahertz spectroscopy
}

Porte, Henrik; Jepsen, Peter Uhd; Daghestani, N.; Rafailov, E.U.; Turchinovich, Dmitry

Published in:

Applied Physics Letters

Link to article, DOI:

$10.1063 / 1.3158958$

Publication date:

2009

Document Version

Publisher's PDF, also known as Version of record

Link back to DTU Orbit

Citation (APA):

Porte, H., Jepsen, P. U., Daghestani, N., Rafailov, E. U., \& Turchinovich, D. (2009). Ultrafast release and capture of carriers in InGaAs/GaAs quantum dots observed by time-resolved terahertz spectroscopy. Applied Physics Letters, 94(26), 262104. https://doi.org/10.1063/1.3158958

\section{General rights}

Copyright and moral rights for the publications made accessible in the public portal are retained by the authors and/or other copyright owners and it is a condition of accessing publications that users recognise and abide by the legal requirements associated with these rights.

- Users may download and print one copy of any publication from the public portal for the purpose of private study or research.

- You may not further distribute the material or use it for any profit-making activity or commercial gain

- You may freely distribute the URL identifying the publication in the public portal 


\title{
Ultrafast release and capture of carriers in InGaAs/GaAs quantum dots observed by time-resolved terahertz spectroscopy
}

\author{
H. P. Porte, ${ }^{1}$ P. Uhd Jepsen, ${ }^{1}$ N. Daghestani, ${ }^{2}$ E. U. Rafailov ${ }^{2}$ and D. Turchinovich ${ }^{1, a)}$ \\ ${ }^{1}$ DTU Fotonik - Department of Photonics Engineering, Technical University of Denmark, \\ DK-2800 Kgs. Lyngby, Denmark \\ ${ }^{2}$ School of Engineering, Physics and Mathematics, University of Dundee, Dundee DD1 4HN, \\ United Kingdom
}

(Received 7 May 2009; accepted 4 June 2009; published online 29 June 2009)

\begin{abstract}
We observe ultrafast release and capture of charge carriers in InGaAs/GaAs quantum dots in a room-temperature optical pump-terahertz probe experiment sensitive to the population dynamics of conducting states. In case of resonant excitation of the quantum dot ground state, the maximum conductivity is achieved at approximately 35 ps after photoexcitation, which is assigned to release of carriers from the quantum dots. When exciting carriers into the conduction band of the barriers, depletion of the conductivity via carrier capture into the quantum dots with a few picosecond pump fluence-dependent time constant was observed. (C) 2009 American Institute of Physics.
\end{abstract}

[DOI: 10.1063/1.3158958]

Understanding of ultrafast carrier dynamics in semiconductor quantum dots (QDs) is important for operation of optoelectronic devices such as QD lasers, ${ }^{1,2}$ QD infrared photodetectors, ${ }^{3}$ and QD semiconductor saturable absorber mirrors (QD SESAMs). ${ }^{4}$

Ultrafast dynamics in QDs was intensively studied in the past using ultrafast optical methods such as timeresolved photoluminescence, ${ }^{5,6}$ and optical pump-probe spectroscopy. ${ }^{7,8}$ Here we observe the carrier release and capture dynamics in $\mathrm{In}_{0.5} \mathrm{Ga}_{0.5} \mathrm{As} / \mathrm{GaAs}$ QDs, using an optical pump-terahertz probe technique. In such an experiment, an ultrafast optical excitation creates a population of electrons and holes, and the broadband terahertz probe pulse subsequently senses the change in the conductivity of the sample after the optical excitation. ${ }^{9}$ Terahertz pulses are strongly sensitive to the presence of conducting carriers, whereas immobile carriers, such as those confined in the QDs, do not contribute significantly to the terahertz loss. ${ }^{8,10-12}$

Our experiment was driven by a regenerative Ti:Sapphire femtosecond amplifier, operating at a repetition rate of $1 \mathrm{kHz}$, and generating $45 \mathrm{fs}$ pulses at $800 \mathrm{~nm}$ central wavelength. A part of the amplifier output was used as a pump pulse: either directly for excitation of the barrier states of the QDs at $800 \mathrm{~nm}$; or after frequency conversion in an optical parametric amplifier to $1024 \mathrm{~nm}$, for resonant excitation of the QD ground state (GS), as shown in Fig. 1(a). Another part of the amplifier output was used to power the nonlinear crystal-based terahertz time-domain spectrometer, with a sample positioned at its focal point. ${ }^{9}$ The terahertz probe pulses had a duration of approximately $300 \mathrm{fs}$ and had a useful spectrum of $0.5-2.5 \mathrm{THz}$, similar to the spectrometer used in Ref. 13. All our measurements were performed at room-temperature.

Our sample was a QD SESAM, grown by molecular beam epitaxy. It had 15 layers of $\mathrm{In}_{0.5} \mathrm{Ga}_{0.5} \mathrm{As} / \mathrm{GaAs}$ QDs separated by GaAs spacers, grown on top of a $\mathrm{Al}_{0.9} \mathrm{Ga}_{0.1} \mathrm{As} / \mathrm{GaAs}$ Bragg reflector. The total thickness of QD and spacer region was $611 \mathrm{~nm}$. The sample was grown

${ }^{a)}$ Electronic mail: dmtu@fotonik.dtu.dk. on a 0.5 -mm-thick semi-insulating GaAs substrate. The schematic of the sample is shown in Fig. 1(b).

Ultrafast processes such as carrier capture, release, and recombination are schematically shown in Fig. 1(a), exemplified by electrons in the conduction band. Also important for the ultrafast carrier dynamics are processes such as intra-
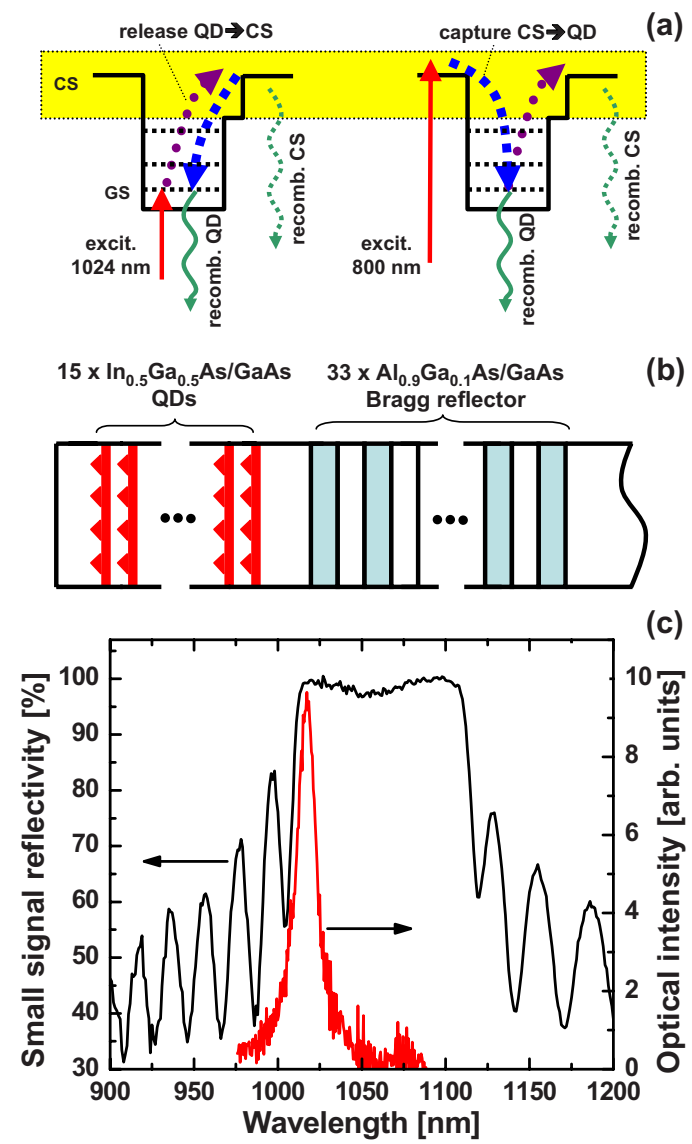

FIG. 1. (Color online) (a) Carrier release and capture processes in photoexcited QDs. CS - conducting state, and GS-QD GS. (b) Schematic of InGaAs/GaAs QD SESAM sample studied in this work. (c) Small signal reflectivity and room-temperature optical emission spectra at $800 \mathrm{~nm}$ excitation of the studied QD SESAM. 
dot carrier relaxation, carrier-carrier and carrier-phonon scattering, and interplay between the carriers in QD and the wetting layer (WL) states. These processes are temperature- and carrier density-dependent. ${ }^{7,14}$ In our experiments, the population of conducting states (CS), consisting of the WL and barrier states, was monitored after optical excitation by measuring the relative transmission $-\Delta T / T_{0}$ of the terahertz probe pulse through the photoexcited sample. Here, $T_{0}$ is the terahertz transmission through the sample before photoexcitation. The measured time dependencies of $-\Delta T / T_{0}$ are then converted into a photoinduced terahertz-frequency-integrated sheet conductivity $\Delta \sigma_{\square, \mathrm{THz}} \propto\left[\mu_{e} n_{e}+\mu_{h} n_{h}\right]$, where $\mu_{e, h}$ and $n_{e, h}$ are the mobility and concentration of electrons and holes, respectively. ${ }^{15}$ Both optical pump and terahertz probe pulses were incident on the sample at normal incidence. Therefore, the in-plane conductivity of the photoexcited sample was probed with the terahertz pulse. The energetic range of the probed CS (for the case of conduction band) is marked in yellow in Fig. 1(a). We note here that in our experiments we were not able to distinguish between the carriers confined in the WLs and the carriers present in the barriers, since both of these states are conducting in the plane of the sample. For the same reason, we were not able to distinguish between conductive electrons and holes. However, the electron contribution to the sample conductivity will be dominating, owing to their higher mobility.

The small-signal reflectivity spectrum of the studied QD SESAM, as well as the room-temperature optical emission spectrum at $800 \mathrm{~nm}$ excitation, indicating the energetic position of the QD GS are shown in Fig. 1(c). The Bragg reflector has a 100-nm-wide stop band centered at $1060 \mathrm{~nm}$. The emission spectrum is centered at a wavelength of $1017 \mathrm{~nm}$. The $1024 \mathrm{~nm}$ optical pump pulse has a full width at half maximum bandwidth of $45 \mathrm{~nm}$, ensuring good overlap with the QD GS in the case of its resonant excitation. The Bragg reflector blocked the propagation of the $1024 \mathrm{~nm}$ pump pulse further into the thick GaAs substrate of the sample, thus preventing free-carrier generation in GaAs by two-photon absorption (TPA). This was very important, since the TPAgenerated free-carriers in a thick GaAs substrate would easily obscure the signal from the carriers released from the QDs at the resonant GS excitation.

In order to observe carrier release from the QD GS to the $\mathrm{CS}$, we performed resonant optical excitation of the QD GS at a wavelength of $1024 \mathrm{~nm}$, with pump fluences in the range 2.1-22.8 $\mu \mathrm{J} / \mathrm{cm}^{2}$. As seen in Fig. 2(a), we observe a noninstantaneous rise in the sample conductivity, reaching its maximum at approximately 35 ps after photoexcitation, followed by a slow decay due to recombination in barriers and WLs. The maximum of $\Delta \sigma_{\square, \mathrm{THz}}$ as a function of pumping fluence exhibits a sublinear behavior as plotted in Fig. 2(b), which suggests that the photoexcitation mechanism leading to supply of carriers to the CS has a one-photon saturable nature (assuming constant carrier mobility), as expected for resonant excitation of the QD GS in this pump fluence range. Fit of this dependency with the saturable absorption ${ }^{16}$ function $\propto F_{p} \exp \left[-F_{p} / F_{s}\right]$, where $F_{p}$ is the pump fluence and $F_{s}$ is the saturation fluence, revealed $F_{s}=40 \mu \mathrm{J} / \mathrm{cm}^{2}$. This is in reasonable agreement with the value of $F_{s}=25 \mu \mathrm{J} / \mathrm{cm}^{2}$ observed in Ref. 4 for a similar QD-based structure. Also in Ref. 4, no TPA-driven carrier generation in the QDs was observed at resonant QD GS excitation with pumping flu-
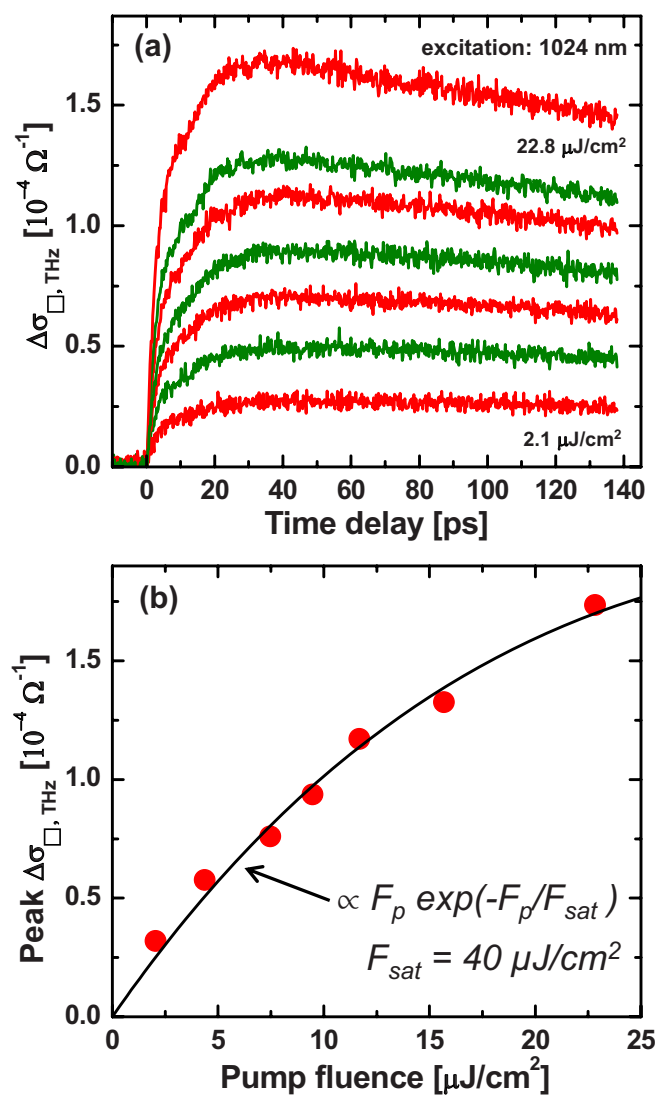

FIG. 2. (Color online) (a) Dynamics of photoinduced sheet conductivity $\Delta \sigma_{\square, \mathrm{THz}}$ at resonant optical excitation of QD GS at $1024 \mathrm{~nm}$, with the pump fluence 2.1-22.8 $\mu \mathrm{J} / \mathrm{cm}^{2}$. (b) Maximum of $\Delta \sigma_{\square, \mathrm{THz}}$ traces as a function of pump fluence for $1024 \mathrm{~nm}$ excitation. Solid line: Fit to the saturable absorption function with saturation fluence $F_{s}=40 \mu \mathrm{J} / \mathrm{cm}^{2}$.

ences up to $1 \mathrm{~mJ} / \mathrm{cm}^{2}$, which agrees well with our observations.

In order to observe the carrier capture dynamics, the carriers were excited directly into the barrier states of the conduction band with $800 \mathrm{~nm}$ excitation. In Fig. 3(a), the corresponding time dependencies of $\Delta \sigma_{\square, \mathrm{THz}}$ are shown for the pump fluences in the range $0.04-1.18 \mu \mathrm{J} / \mathrm{cm}^{2}$. A nearinstantaneous rise in photoinduced conductivity is observed, followed by a fast decay and by a very long-lived contribution. It is reasonable to attribute the fast decay to capture of the carriers into the QDs, ${ }^{8,10,11}$ as supported by the slower initial conductivity decay dynamics observed in a bulk GaAs sample [dashed line in Fig. 3(a)]. A very long-lived contribution, persistent even after an experimental cycle of $1 \mathrm{~ms}$ in case of the strongest excitation, is most likely due to spatially separated electrons and holes via $\Gamma-X$ transfer $^{17}$ in the type-II $\mathrm{Al}_{0.9} \mathrm{Ga}_{0.1} \mathrm{As} / \mathrm{GaAs}$ SESAM Bragg reflector, which was accessible to the pump light at this wavelength. This very longlived contribution leads to a small nonzero background in measured photoconductivities at time delays $t<0$. In the QD SESAM data shown in Fig. 3(a), this background has been subtracted. A small kink in the $800 \mathrm{~nm}$ traces at around $22 \mathrm{ps}$ is an experimental artifact arising from a double reflection in a 2-mm-thick pump beam attenuator during the measurements. The initial rise of $\Delta \sigma_{\square, \mathrm{THz}}$ shows linear dependency on excitation fluence (not shown here), as expected for a one-photon excitation process.

In order to estimate the carrier capture time, we have measured the time it takes for the photoinduced conductivity 

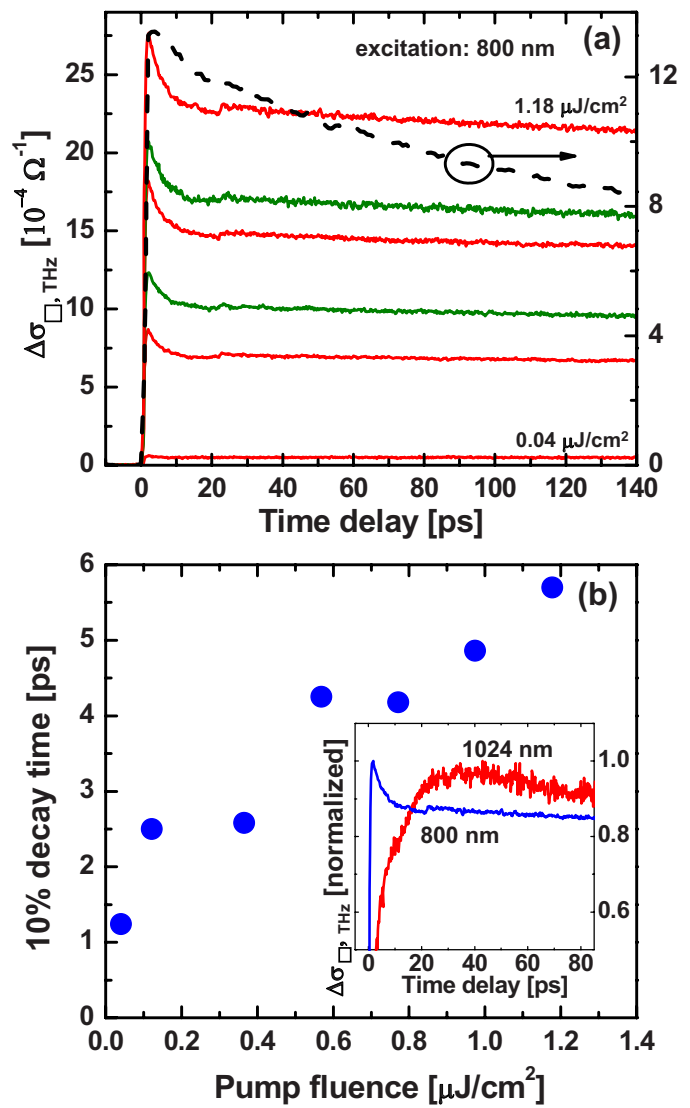

FIG. 3. (Color online) (a) Solid lines: Dynamics of photoinduced sheet conductivity of a QD SESAM $\Delta \sigma_{\square, \mathrm{THz}}$ at optical excitation of barriers at $800 \mathrm{~nm}$, with the pump fluence varied between $0.04-1.18 \mu \mathrm{J} / \mathrm{cm}^{2}$. Dashed line: $\Delta \sigma_{\square, \mathrm{THz}}$ for a bulk GaAs sample at $800 \mathrm{~nm}$ excitation with the pump fluence of $0.9 \mu \mathrm{J} / \mathrm{cm}^{2}$; (b) $10 \%$ decay time constant of the QD SESAM $\Delta \sigma_{\square, \mathrm{THz}}$ traces in (a), as a function of $800 \mathrm{~nm}$ pump fluence. Inset: Normalized $\Delta \sigma_{\square, \mathrm{THz}}$ traces for QD GS and barrier excitation with the pump wavelength and fluence of $1024 \mathrm{~nm}$ and $22.8 \mu \mathrm{J} / \mathrm{cm}^{2}$, and $800 \mathrm{~nm}$ and $1.18 \mu \mathrm{J} / \mathrm{cm}^{2}$, respectively.

to decay by $10 \%$ in respect to its maximum. A $10 \%$ benchmark was chosen because the maximum value of fast decay in our data only reaches $20 \%$ due to the presence of the long-lived component. This $10 \%$ time constant demonstrates near-linear growth from 1.2 to $5.7 \mathrm{ps}$ with increase in pump fluence, as shown in Fig. 3(b). We attribute this growth in carrier capture time constant to filling of the QD (trap) states at stronger excitation, which is in accordance with the observations made in Refs. $10,11,15$, and 18 . We note here that our observations are in contrast with the speeding up of carrier capture with increase in pump fluence, mentioned in Refs. 7 and 8. In Ref. 7, the population dynamics of only the GS of the QD was observed. However, one may speculate that the suggested trapping mechanism-electron-electron scattering-may also provide for the scattering of the carriers from higher-lying QD states back into the CS, thus slowing down the decay dynamics of the conductivity (which was not monitored in that experiment). The reason for the disagree- ment between our observations and those in Ref. 8 is not clear at this point.

In conclusion, we were able to demonstrate the difference in sample conductivity dynamics depending on whether the QD GS or the barrier states are optically excited [see inset of Fig. 3(b)]. The observed dynamics can be explained by the dominating carrier release or carrier capture processes, respectively. The $e 1 h 1-\mathrm{WL}$ energy gap was measured to be $252 \mathrm{meV}$ in a similar structure, shared approximately in $60 / 40$ proportion between the electron and hole states. ${ }^{7}$ This largely exceeds $k_{B} T=26 \mathrm{meV}$ at room-temperature for both electron and hole GS-CS transitions. However, the large difference in densities of states between the QD GS and CS should increase the probability of GS-CS carrier release. Our further investigations will be focused on temperature- and carrier density-dependent measurements that would allow us to better understand the ultrafast carrier dynamics in QDs.

We are grateful to the Danish Advanced Technology Foundation (HTF), and EU FP7 Programme (FAST-DOT), Grant No. 224338, for financial support; and to D. A. Livshits (Innolume GmbH), K. Iwaszczuk, D. G. Cooke, and J. M. Hvam (DTU Fotonik) for valuable assistance and discussions.

${ }^{1}$ N. Kirstaedter, O. G. Schmidt, N. N. Ledentsov, D. Bimberg, V. M. Ustinov, A. Yu. Egorov, A. E. Zhukov, M. V. Maximov, P. S. Kop'ev, and Zh. I. Alferov, Appl. Phys. Lett. 69, 1226 (1996).

${ }^{2}$ E. U. Rafailov, M. A. Cataluna, and W. Sibbett, Nat. Photonics 1, 395 (2007)

${ }^{3}$ H. C. Liu, M. Gao, J. McCaffrey, Z. R. Wasilewski, and S. Fafarda, Appl. Phys. Lett. 78, 79 (2001).

${ }^{4}$ A. A. Lagatsky, F. M. Bain, C. T. A. Brown, W. Sibbett, D. A. Livshits, G. Erbert, and E. U. Rafailov, Appl. Phys. Lett. 91, 231111 (2007).

${ }^{5}$ D. Morris, N. Perret, and S. Fafard, Appl. Phys. Lett. 75, 3593 (1999).

${ }^{6}$ K. Gündoğdu, K. C. Hall, T. F. Boggess, D. G. Deppe, and O. B. Shchekin, Appl. Phys. Lett. 85, 4570 (2004).

${ }^{7}$ T. Müller, F. F. Schrey, G. Strasser, and K. Unterrainer, Appl. Phys. Lett. 83, 3572 (2003).

${ }^{8}$ D. A. Yarotski, R. D. Averitt, N. Negre, S. A. Crooker, A. J. Taylor, G. P. Donati, A. Stintz, L. F. Lester, and K. J. Malloy, J. Opt. Soc. Am. B 19, 1480 (2002)

${ }^{9}$ R. Huber, F. Tauser, A. Brodschelm, M. Bichler, G. Abstreiter, and A. Leitenstorfer, Nature (London) 414, 286 (2001).

${ }^{10}$ D. Turchinovich, K. Pierz, and P. Uhd Jepsen, Phys. Status Solidi C 0, 1556 (2003).

${ }^{11}$ D. G. Cooke, F. A. Hegmann, Yu. I. Mazur, W. Q. Ma, X. Wang, Z. M. Wang, G. J. Salamo, M. Xiao, T. D. Mishima, and M. B. Johnson, Appl. Phys. Lett. 85, 3839 (2004).

${ }^{12}$ R. P. Prasankumar, A. Scopatz, D. J. Hilton, A. J. Taylor, R. D. Averitt, J. M. Zide, and A. C. Gossard, Appl. Phys. Lett. 86, 201107 (2005).

${ }^{13}$ M. Schall and P. Uhd Jepsen, Opt. Lett. 25, 13 (2000).

${ }^{14}$ T. R. Nielsen, P. Gartner, and F. Jahnke, Phys. Rev. B 69, 235314 (2004).

${ }^{15}$ K. P. H. Lui and F. A. Hegmann, Appl. Phys. Lett. 78, 3478 (2001).

${ }^{16} \mathrm{M}$. Haiml, R. Grange, and U. Keller, Appl. Phys. B: Lasers Opt. 79, 331 (2004).

${ }^{17}$ J. Feldmann, J. Nunnekamp, G. Peter, E. Göbel, J. Kuhl, K. Ploog, P. Dawson, and C. T. Foxon, Phys. Rev. B 42, 5809 (1990).

${ }^{18} \mathrm{P}$. Uhd Jepsen, W. Schairer, I. H. Libon, U. Lemmer, N. E. Hecker, M. Birkholz, K. Lips, and M. Schall, Appl. Phys. Lett. 79, 1291 (2001). 